\title{
PHYSICS OF HOT ELECTRON TRANSPORT IN SEMICONDUCTORS
}


This page is intentionally left blank 


\title{
PHYSICS OF
}

\section{HOT ELECTRON TRANSPORT} IN SEMICONDUCTORS

\author{
edited by \\ C. S. Ting \\ Texas Center for Superconductivity \\ University of Houston
}




\author{
Published by \\ World Scientific Publishing Co. Pte. Ltd. \\ P O Box 128, Farrer Road, Singapore 9128 \\ USA office: Suite 1B, 1060 Main Street, River Edge, NJ 07661 \\ UK office: 73 Lynton Mead, Totteridge, London N20 8DH
}

\title{
PHYSICS OF HOT ELECTRON TRANSPORT IN SEMICONDUCTORS
}

Copyright @ 1992 by World Scientific Publishing Co. Pte. Ltd.

All rights resenved. This book, or parts thereof, may not be reproduced in any form or by any means, electronic or mechanical, including photocopying, recording or any information storage and retrieval system now known or to be invented, without written permission from the Publisher.

ISBN $981-02-1008-6$

Printed in Singapore by JBW Printers \& Binders Pte. Ltd. 


\section{PREFACE}

The development of submicrometer semiconductor devices during the past decade has underscored the need for a better theoretical understanding of carrier transport in these small sized systems. Even a few volts applied across the device can lead to a rather high electric field on the order of $10^{4} \mathrm{~V} / \mathrm{cm}$. In these circumstances, the relationship between current and voltage is no longer linear, and the statistical temperature of the charge carriers becomes higher than that of the heat bath. Thus, the carrier dynamics under a strong electric field are characterized as "hot electron transport" Since the first survey on this subject by E. M. Conwell in her review High Field Transport in Semiconductors (New York: Academic Press, 1967), where the experimental data were analyzed with the phenomenological Boltzmann equation, many new experimental techniques and theoretical methods have been developed or proposed to study hot electron transport in various semiconductors. In particular, Monte Carlo simulation methods have been introduced to solve the Boltzmann equation numerically. A useful review of such developments can be found in the book Hot Electron Transport in Semiconductors edited by L. Reggiani (New York: Springer-Verlag, 1985). However, in the present volume, we shall review primarily analytical approaches developed since 1984 for treating various kinds of hot electron transport problems.

The balance equation method, originally proposed by X. L. Lei and myself in 1984 for the purpose of investigating steady state hot electron transport, has subsequently been extended to study thermal noise, time dependent transport, and other subjects relating to charge carriers in strong electric fields. The predicted theoretical results are in good agreement with both experimental measurements and Monte Carlo numerical simulations. Much of the simplicity of the mathematical structure of the balance equation approach arises in conjunction with its effectiveness in dealing with many-electron systems having strong inter-carrier collisions, and it is particularly powerful in this respect. Extensive reviews of this method are given by the first two articles, which deal with it in the absence and presence of a magnetic field. When hot electrons are confined to a quantum well, the proper treatment of the electron-phonon-coupling involving phonons which propagate in three dimensions requires special care, because phonon heating produces a bottleneck in the rate energy transfer from electrons to phonons. This subject is carefully reviewed in Article 3. During the past decades, the intra-collisional field effect (ICFE), which has been promoted as a major ingredient of quantum transport theory, has drawn tremendous theoretical efforts in the attempt to understand its influence on hot electron transport (see Section 16, Article 1). Nevertheless, all the numerical calculations to date seem to indicate that this effect yields almost no correction to steady state transport up to a field strength of $10^{7} \mathrm{~V} / \mathrm{cm}$ except possibly minor modifications to the classical overshoot behavior within a time scale $t>10^{12} \mathrm{sec} .(\sim$ electron-phonon scattering time) in the transient region respond to a step electric field. Within the framework of the balance equation formulation, the intra-collisional field term is simply embedded in the structure of the memory 
function wherein the drift velocity of the charge carriers is taken in its time dependent form - accelerated by the electric field, even with the neglect of contributions from the electron-phonon interaction and other scattering mechanisms. This feature will be discussed in Article 4, where the memory effect is demonstrated to be very important for investigating nonlinear high frequency conductivity. The method of nonequilibrium statistical operators (NSO), developed by Zubarev in the early sixties, has subsequently been employed by Kalashnikov in 1970 to study hot electron transport. In Article 5, it will be shown that had the problem been done correctly, the results obtained from NSO would reduce exactly to those of the balance equation method as described in Article 1. In this connection, I wish to emphasize that Articles 1 to 5 in this book assume charge carriers with strong carrier-carrier collisions. These collisions may originate directly from Coulomb interaction and/or indirectly from phonon mediated electron-electron interaction, with the strength of such interaction mandating a relatively high carrier density. On the other hand, for the case of very low carrier density, the charge carriers are far apart and collisions between carriers are negligible. In this situation, the transport problem needs to be treated in terms of single carrier transport, which will be discussed in Article 6 using the nonequilibrium Green's function techniques and the Feynman path integral theory. At this stage, it is of interest to note that the linear isothermal (I) impurity resistivity derived from the balance equation method outlined in Articles 1 to 5 yields the expression $\rho_{i}^{I}=\frac{m}{N e^{2}}\left\langle\frac{1}{\tau}\right\rangle$, with $\frac{1}{\tau}$ and $\langle\cdots\rangle$ as defined in Equations (15.38) and (15.39) of Article 1. This form is different from the standard adiabatic (A) result based upon the Kubo formula or the Boltzmann equation in the absence of carrier-carrier scattering, wherein $\rho_{i}^{A}=\frac{m}{N e^{2}(\tau)}$. Although $\rho_{i}^{A}$ and $\rho_{i}^{I}$ are practically identical with each other at low temperatures, they are quite different at higher temperatures. The question of which formula should be used to compare with experimental measurements has been the subject of debate in the literature for quite some time. In Article 7, the authors introduce an inter-carrier collision time $\tau_{i n}$ and show that $\rho_{i}^{I}$ and $\rho_{i}^{A}$ are derived respectively under the conditions $\tau_{\text {in }} \ll \tau$ (strong inter-carrier collisions) and $\tau_{\text {in }} \ll \tau$ (weak inter-carrier collisions). Finally, in the last paper, the dynamic conductivity and the shot noise suppression of a double-barrier resonant tunneling system, which is of great current interest (but having no links to the previous articles,) is also reviewed.

Finally, I wish to thank all the contributors to this volume for their cooperation and special efforts in writing their review articles to meet the highest standards of quality in research, as well as pedagogical clarity. Acknowledgement is also due to the Texas Center for Superconductivity at the University of Houston for support in every respect. 


\section{CONTENTS}

Preface

Balance-Equation Approach to Hot-Carrier Transport in Semiconductors

X. L. Lei and N. J. M. Horing

1. Introduction

2. Fundamental Physical Considerations

2.1 Center-of-mass and relative variables of electrons 4

2.2 Center of mass treated as a classical particle 6

2.3 Initial density matrix $\quad 8$

3. Force- and Energy-Balance Equations to Lowest Order in $H_{I}$

3.1 Density matrix and statistical averages to linear order in $H_{I} \quad 10$

3.2 Force balance equation 10

3.3 Energy balance equation 11

4. Steady-State dc Transport in a 3D System 13

4.1 Balance equations for steady state 13

$\begin{array}{ll}4.2 \text { Weak current ohmic resistivity } & 15\end{array}$

4.3 Nonlinear zero-lattice-temperature limit $\quad 17$

4.4 Nondegenerate (Maxwell-Boltzmann) case, acoustic phonon scattering 18

4.5 Optic phonon scattering 20

4.6 Electron cooling $\quad 21$

4.7 Role of dynamic screening $\quad 22$

5. dc Steady State Transport in Quasi-Two-Dimensional Systems and Superlattices 24

5.1 Balance equations for quasi-two-dimensional systems 24

5.2 Hot electron transport in Ga-As/AlGaAs heterojunctions

5.3 Balance equations for quantum-well superlattices 31

6. Transient Transport 37

6.1 Transient balance equations without memory 37

6.2 Memory effects in transient response for the weak current case $\quad 39$

7. High-Frequency Steady State Transport 42

7.1 High-frequency conductivity for small signal $\quad 42$

7.2 Plasmon contribution to high-frequency conductivity in quasi-2D systems and superlattices

$\begin{array}{ll}\text { 7.3 Nonlinear high-frequency conductivity } & 47\end{array}$

8. C. M. Velocity Fluctuations and Related Matters 48

$\begin{array}{ll}\text { 8.1 Langevin-type equation } & 48\end{array}$

8.2 Thermal noise and diffusion $\quad 50$

8.3 Role of velocity fluctuations in drift motion $\quad 55$

9. Several Species of Carriers $\quad 57$

9.1 Type-II superlattices, electron-hole plasma $\quad 57$

9.1.1 Balance equations for a type-II superlattice $\quad 57$

$\begin{array}{ll}\text { 9.1.2 Steady-state nonlinear dc transport } & 59\end{array}$

9.1.3 Negative minority-electron mobility in an electron-hole plasma $\quad 60$

9.1.4 Linear high-frequency transport 62 
9.2 Multivalley semiconductors

9.2.1 Systems of two carrier species with particle interchange 63

9.2.2 Balance equations and Langevin equations 65

9.2.3 Results for dc steady state and transient transport 68

$\begin{array}{ll}\text { 9.2.4 Correlations of the velocity and particle number fluctuations } & 70\end{array}$

10. Nonequilibrium Phonon Phenomena $\quad 72$

$\begin{array}{ll}10.1 \text { Nonequilibrium phonons in 3D bulk systems } & 72\end{array}$

$\begin{array}{ll}10.2 \text { Nonequilibrium phonons in quantum wells and superlattices } & 75\end{array}$

10.3 Effects of nonequilibrium phonon occupation on hot electron transport $\quad 76$

$\begin{array}{ll}10.4 \text { Phonon confinement in semiconductor quantum wells } & 78\end{array}$

11. Balance Equations for Hot-Electron Transport in the Presence

of a Magnetic Field

11.1 Balance equations in the presence of crossed electric and magnetic fields $\quad 80$

11.2 Hot electron magnetoresistivity $\quad 82$

12. Effects of Higher Order Scatterings $\quad 84$

12.1 CTPG formulation of the balance-equation approach 84

$\begin{array}{ll}12.2 \text { Electric field effects on weak localization } & 87\end{array}$

13. Balance Equations for Weakly Nonuniform Systems 91

13.1 Hamiltonian of the small system elements 91

13.2 Rates of change of particle number, momentum and energy 93

13.3 Hydrodynamic balance equations 94

14. Alternative Formulations and Interpretations of the

Balance Equation Approach

14.1 Dielectric interpretation of the impurity-induced force 96

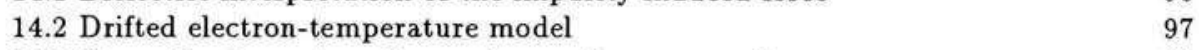

14.3 Generalized quantum Langevin equation approach $\quad 99$

$\begin{array}{ll}\text { 14.4 Nonequilibrium statistical operator method } & 101\end{array}$

15. Isothermal Transport and Balance-Equation Approach 104

$\begin{array}{ll}15.1 \text { Full quantum-mechanical treatment } & 104\end{array}$

15.2 Perturbative expansion and steady-state balance equations $\quad 106$

$\begin{array}{lr}15.3 \text { Isothermal and adiabatic resistivities } & 109\end{array}$

15.4 Balance-equation theories for a single electron system 112

16. Balance Equations for General Systems with Intracollisional Field Effect $\quad 113$

16.1 Force and energy-loss-rate with intracollisional field effect 113

16.2 General system formulations without intracollisional field effect $\quad 115$

$\begin{array}{ll}\text { 16.3 Evaluation of the intracollisional field effect } & 117\end{array}$

17. Recent Developments of the Balance-Equation Theory 120

$\begin{array}{ll}\text { 17.1 Distribution function in the balance-equation theory } & 120\end{array}$

17.2 Improved balance-equation calculation for GaAs/AlGaAs heterojunctions - a step towards device simulation

17.3 Balance equations for single arbitrary energy band and application to superlattice miniband transport

Recent Developments in Magnetotransport Theory

N. J. M. Horing, H. L. Cui, and X. L. Lei

1. Introduction: Balance Equation Transport Theory in Magnetic Fields 
2. Magnetotransport in Bulk Semiconductors Revisited: Linear and Nonlinear

2.1 Longitudinal geometry

2.2 Transverse geometry

3. Magnetotransport in Microstructures $\quad 151$

$\begin{array}{ll}3.1 \text { Type-I superlattice } & 151\end{array}$

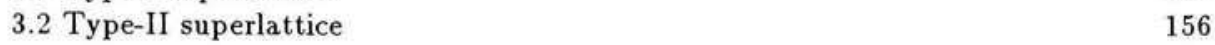

4. Concluding Remarks and Summary 163

Dedication $\quad 165$

$\begin{array}{ll}\text { References } & 166\end{array}$

Effect of Nonequilibrium Phonons on the Electron Relaxation and Transport 171 M. Lax and W. Cai

1. The Problem 172

2. Our Resolution 172

2.1 Quasi-equilibrium $\quad 173$

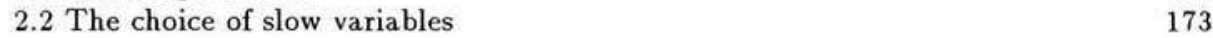

2.3 Description of the Hamiltonian 173

2.4 Form of the equations of motion $\quad 174$

$\begin{array}{ll}2.5 \text { The shape of the packet } & 175\end{array}$

$\begin{array}{ll}2.6 \text { Further simplifications } & 176\end{array}$

$\begin{array}{ll}2.7 \text { The reduced equations } & 177\end{array}$

2.8 Energy-loss-rate in steady state case $\quad 178$

$\begin{array}{ll}2.9 \text { Time-dependent relaxation } & 181\end{array}$

3. Further Applications of the Theory 182

$\begin{array}{ll}3.1 \text { Hot electron transport } & 182\end{array}$

3.2 Relaxation of the electron-hole plasma $\quad 185$

3.3 Electron-hole transport and negative mobility 187

4. Other Theoretical Works 192

4.1 Monte Carlo simulation of nonequilibrium phonon effect 192

$\begin{array}{lr}4.2 \text { Effect of LO-phonon renormalization } & 195\end{array}$

$\begin{array}{ll}\text { 5. Summary } & 199\end{array}$

$\begin{array}{ll}\text { Appendix } & 199\end{array}$

Nonlinear Transport of Electrons under a

Strong High Frequency Electric Field in Semiconductors 203

W. Cai and M. Lax

1. Introduction 203

$\begin{array}{ll}1.1 \text { Previous theories } & 205\end{array}$

1.2 Experiments 208

2. Transport Equations under a Strong High Frequency Electric Field 210

$\begin{array}{ll}2.1 \text { Hamiltonian } & 210\end{array}$

2.2 Equations for drift velocity of electrons $\quad 211$

$\begin{array}{ll}2.3 \text { Equation for distribution of hot-electrons } & 216\end{array}$

3. Linear High Frequency Conductivity 219

$\begin{array}{lr}3.1 \text { Cooling electron case } & 219\end{array}$

$\begin{array}{ll}3.2 \text { Hot electron case } & 220\end{array}$ 
4. Nonlinear HF Conductivity

5. Discussion

Appendix

Nonequilibrium Statistical Operator in Hot-Electron Transport Theory D. Y. Xing and M. Liu

1. Introducton

2. Hamiltonian

3. Nonequilibrium statistical operator

4. Balance Equations for Momentum and Energy

5. Application to Multivalley Semiconductors

6. Extensions to Transient Hot-Electron Transport

7. Concluding Remarks

References

Path Integral Study of Polaron Transport under High Electric Field Z. B. $S u$

1. Introduction

2. Nonequilibrium Green's Function Description of the Drift and Fluctuation

3. Steady State Transport

4. Energy Balance Equation 264

5. Numerical Results 265

$\begin{array}{ll}\text { Appendix A } & 270\end{array}$

$\begin{array}{ll}\text { Appendix B } & 270\end{array}$

$\begin{array}{ll}\text { Appendix C } & 271\end{array}$

$\begin{array}{ll}\text { Appendix D } & 271\end{array}$

$\begin{array}{ll}\text { Appendix E } & 272\end{array}$

$\begin{array}{ll}\text { References } & 273\end{array}$

Impurity Resistivity under Thermalized Condition $\quad 275$

C. S. Ting and L. Y. Chen

1. Introduction 275

2. Quantum Mechanical Treatment of Center of Mass Variables 277

3. Vanishing Current and Correct Finite Result of Adiabatic Resistivity 283

4. Consideration of the Exact Noncanonical Relative Electron Commutation Relations

5. Boltzmann Equation in Relaxation Time Approximation 288

6. Summary and Conclusions 289

Appendix $\quad 290$

References $\quad 293$ 
Nonequilibrium Green's Function Approach to Dynamic Properties of Resonant-Tunneling through Double Barrier Structures

$$
\text { L. Y. Chen and C. S. Ting }
$$

1. Introduction

2. General Description 297

3. Transient Response $\quad 300$

4. Frequency Response 303

5. Noise Characteristics 308

6. Concluding Remarks 312

References 312 